\title{
Comparison of Thoracic Radiography and Computed Tomography in Calves with Naturally Occurring Respiratory Disease
}

\author{
Jennifer Fowler ${ }^{1}$, Susanne M. Stieger-Vanegas ${ }^{1 *}$, Jorge A. Vanegas ${ }^{1}$, Gerd Bobe $^{2,3}$ \\ and Keith P. Poulsen ${ }^{4}$

\begin{abstract}
'Department of Clinical Sciences, College of Veterinary Medicine, Oregon State University, Corvallis, OR, United States, United States, ${ }^{3}$ Linus Pauling Institute, Oregon State University, Corvallis, OR, United States, ${ }^{4}$ Wisconsin Veterinary Diagnostic Laboratory, Department of Medical Sciences, School of Veterinary Medicine, University of Wisconsin, Madison, WI, United States
\end{abstract} \\ ${ }^{2}$ Department of Animal and Rangeland Sciences, College of Agricultural Sciences, Oregon State University, Corvallis, OR,
}

OPEN ACCESS

Edited by: Mário Ginja, Universidade de Trás-os-Montes e Alto Douro (UTAD), Portugal

Reviewed by: José Manuel Gonzalo Orden, Universidad de León, Spain Christopher R. Lamb, Royal Veterinary College, United Kingdom

${ }^{*}$ Correspondence: Susanne M. Stieger-Vanegas susanne.stieger@oregonstate.edu

This study was performed at the Lois Bates Acheson Veterinary Teaching Hospital, Oregon State University, Corvallis, OR, USA.

Specialty section: This article was submitted to Veterinary Imaging, a section of the journal Frontiers in Veterinary Science

Received: 24 January 2017 Accepted: 12 June 2017

Published: 06 July 2017

Citation:

Fowler J, Stieger-Vanegas SM, Vanegas JA, Bobe $G$ and Poulsen KP (2017) Comparison of Thoracic

Radiography and Computed Tomography in Calves with Naturally Occurring Respiratory Disease.

Front. Vet. Sci. 4:101. doi: 10.3389/fvets.2017.00101
Objective: To evaluate the severity and extent of lung disease using thoracic computed radiography (CR) compared to contrast-enhanced multi-detector computed tomography (MDCT) of the thorax in calves with naturally occurring respiratory disease and to evaluate the feasibility and safety of performing contrast-enhanced thoracic multi-detector MDCT examinations in sedated calves. Furthermore, to evaluate if combining CR or MDCT with respiratory scoring factors will improve prediction of the chronicity of pulmonary disease in calves.

Animals: Thirty Jersey heifer calves ranging in age between 25 and 89 days with naturally occurring respiratory disease.

Procedures: All calves were evaluated via thoracic CR and contrast-enhanced MDCT. All calves were euthanized immediately following thoracic MDCT and submitted for necropsy. Imaging and histopathology results were compared with each other.

Results: Thoracic MDCT was superior for evaluation of pneumonia in calves due to the lack of summation in all areas of the lungs. Intravenously administered sedation provided an adequate plane of sedation for acquiring MDCT images of diagnostic quality, without the need for re-scanning. A diagnosis of pneumonia was made with equal rate on both thoracic $\mathrm{CR}$ and MDCT. Although mild differences in classification of lung pattern and extent of lung disease were seen when comparing an experienced and a less experienced evaluator, the overall differences were not statistically significant. The best intra- and inter-observer agreement was noted when evaluating the cranioventral aspects of the lungs in either modality. Clinical respiratory scoring is inadequate for diagnosing chronicity of pneumonia in calves with naturally occurring pneumonia.

Conclusion and clinical importance: Both imaging modalities allowed diagnosis of pneumonia in calves. The cranial ventral aspects of the lungs were most commonly affected. Thoracic CR and MDCT provided similar diagnostic effectiveness in diagnosing pneumonia. However, MDCT provided better assessment of subtle details, which may be otherwise obscured due to summation artifact.

Keywords: computed radiography, multi-detector computed tomography, calf, naturally occurring respiratory disease, pneumonia 


\section{INTRODUCTION}

Respiratory disease in cattle is the most prevalent cause of morbidity and mortality in beef feedlots, and the most common cause of morbidity in weaned dairy calves (1). Early diagnosis of disease is essential to reduce the risk of disease propagation and to decrease the financial costs from treatment and loss of animals. Furthermore, early diagnosis promotes good quality of life for the patient, improves response to treatment, and decreases the likelihood of prolonged morbidity following juvenile pneumonias $(2,3)$.

Calves with respiratory disease often present with fever, cough, and tachypnea. Auscultation is commonly used to further evaluate the lungs. However, several of these clinical findings have been proven unreliable to diagnose pneumonia in calves. As such, diagnosis of bovine respiratory disease remains a diagnostic challenge.

Thoracic radiography is the most commonly used test of choice to evaluate for pneumonia in human and veterinary patients. Radiography of the thorax of large animals can be limited to having only lateral projections of the thorax available for interpretation and by inadequate penetration due to the size of large animals and equipment limitations; however, its practicality including its use in field conditions, low cost, and widespread availability make it one of the most common used techniques to evaluate the thorax in large animals with respiratory disease (4-6). Computed tomography (CT) of the thorax is considered the modality of choice to evaluate for the presence of lung disease in human patients and is increasingly used in veterinary patients (7). Thoracic CT has proven to be helpful for evaluating the extent of pulmonary disease and provides better anatomic detail due to a lack of superimposition of anatomical structures (8). The use of CT in calves is often limited by the cost and lack of availability of a CT scanner close to a calf rearing operation. Furthermore, CT examinations of the thorax are commonly performed under general anesthesia with endotracheal intubation to allow using breath-hold techniques, which decrease the risk of respiratory motion $(9,10)$. However, calves with severe respiratory impairment due to pneumonia often have an increased anesthetic risk. Although CT scanning protocols have been described for awake and sedated patients including for normal cats and cats with upper airway obstruction and intrathoracic disease, dogs with acute abdominal signs, and dogs with traumatic pelvic fractures, as well as healthy sedated foals neither has been evaluated in calves to date $(11-15)$.

Our objective was to compare the severity and extent of lung disease using computed radiography (CR) compared to contrastenhanced multi-detector CT (MDCT) of the thorax and further determine the feasibility and safety of performing thoracic MDCT examinations in sedated calves with naturally occurring respiratory disease. We hypothesized that thoracic CR would be sufficient to diagnose lung disease in calves; but that MDCT in sedated calves could be safely performed and would provide more

Abbreviations: CdD, caudodorsal; CdV, caudoventral; $\mathrm{CR}$, computed radiography; $\mathrm{CrD}$, craniodorsal; CrV, cranioventral; CT, computed tomography; HU, Hounsfield units; MDCT, multi-detector computed tomography; ROI, region of interest. information about the extent of lung involvement. We further wanted to evaluate if combining CR or MDCT with respiratory scoring factors will improve prediction of the chronicity of pulmonary disease in calves.

\section{MATERIALS AND METHODS}

\section{Study Population}

Thirty privately owned pre-weaned Jersey heifer calves with naturally occurring respiratory disease ranging in age between 25 and 89 days ( $50.5 \pm 18.8$ days) were included in the study. The calves with respiratory disease were identified by farm workers dedicated to calf health monitoring and treatment using a clinical respiratory disease scoring system (16). On the farm, the calves were grouped based on respiratory disease duration into acute disease (within $24 \mathrm{~h}$ of first signs of clinical pulmonary disease, group 1) and chronic disease. Calves with chronic pulmonary disease had received antibiotic treatment at the onset of clinical pulmonary disease but continued to exhibit clinical signs of pneumonia. Chronic disease calves were subdivided into two groups: group 2 included calves which had received one antibiotic treatment 1 week prior (short-term chronic disease), and group 3 included calves which had received antibiotic treatments 2 weeks and 1 week prior (long-term chronic disease).

To study the effect of naturally occurring respiratory disease duration on imaging findings out of each group (acute, shortterm, and long-term chronic), ten calves were randomly selected and transported to the Lois Bates Acheson Veterinary Teaching Hospital at Oregon State University for the imaging studies. Calves were housed with free choice of water, calf starter, and were fed milk replacer twice daily until the imaging studies were performed. All imaging studies were performed within 24-48-h post respiratory scoring on the farm. The study was approved by the Oregon State University Institutional Animal Care and Use Committee.

\section{Thoracic Computed Radiography}

Standing left to right lateral computed radiography (FCR ClearView CS IIP and Type C IP, Fujifilm Co., Tokyo, Japan) images of the thorax were obtained using $85 \mathrm{kVp}$ and $20-32 \mathrm{mAs}$ at $630 \mathrm{~mA}$. If calves were smaller in size, the exposure was adjusted by reducing the $\mathrm{mAs}$ to 20 and keeping the $\mathrm{mA}$ constant at 630 to ensure optimal image quality. One or up to three radiographs of the thorax were obtained to ensure that all aspects of the lungs were included in the study. All diagnostic images were sent to a designated image storage server for off-line analysis.

\section{Multi-Detector Computed Tomography}

All thoracic CT studies were performed under sedation using a 64-row MDCT scanner (Toshiba Aquilion 64 CT, Toshiba America Medical Systems Inc., Tustin, CA, USA). Prior to the MDCT study, a jugular vein catheter was aseptically placed in each calf. Calves were sedated with a combination of $0.1 \mathrm{mg} / \mathrm{kg}$ butorphanol (Torbugesic ${ }^{\circledR}$, Pfizer, New York, NY, USA), $0.3 \mathrm{mg} / \mathrm{kg}$ ketamine (Ketaset ${ }^{\circledR}$, Pfizer/Boehringer, St. Joseph, MO, USA), and $0.4 \mathrm{mg} / \mathrm{kg}$ xylazine (Anased ${ }^{\circledR}$, Lloyd Laboratories, Shenandoah, 
IA, USA) for the MDCT study. All thoracic MDCT scans were performed with the calves positioned in sternal recumbency. A non-contrast enhanced thoracic MDCT scan followed by a contrast-enhanced MDCT scan was performed in each calf from approximately $10 \mathrm{~cm}$ cranial to the thoracic inlet to the mid-level of the left kidney using the following scan parameters: $0.5-\mathrm{mm}$ collimation, $0.5-\mathrm{mm}$ reconstruction interval, $1 \mathrm{~s}$ tube rotation time, $120 \mathrm{kV}, 400 \mathrm{~mA}$, a pitch factor of 0.828 , and 0 -degree tilt. The contrast-enhanced MDCT scan was performed $60 \mathrm{~s}$ after the start of an intravenous iodinated contrast agent (Isovue 300, Bracco Diagnostics Inc., Princeton, NJ, USA) injection at $1 \mathrm{~mL} /$ kg using a power injector (Empower CTA, Bracco Diagnostics Inc., Princeton, NJ, USA) at a flow rate of $3 \mathrm{~mL} / \mathrm{s}$. The thin collimated MDCT isovolumetric data were used to create transverse, sagittal, and dorsal reconstructed images of the thorax with 3-mm slice thickness. A bone, soft tissue, and lung algorithm was used to create bone, soft tissue, and lung window images. Images were sent to a designated image storage server for later analysis. Following MDCT imaging, the calves were humanely euthanatized with intravenous pentobarbital (Beuthanasia ${ }^{\circledR}-\mathrm{D}$ Special, Schering-Plough Animal Health Corp, Union, NJ, USA) at $0.2 \mathrm{ml} / \mathrm{kg}$ in accordance with the American Veterinary Medical Association guidelines.

\section{Image Evaluation}

All diagnostic imaging studies were evaluated independently by two evaluators (JF and SSV) using a commercially available DICOM viewer software (eFilm, version 3.3.0, Merge Healthcare, Hartland, WI, USA;) 3D images were created for the figures using a commercially available 3D imaging software (Vitrea workstation, software version 6.3.2, Vital Images Inc., Minnetonka, MN, USA). Each thoracic CR and MDCT study of each calf was evaluated independently and separately by each evaluator. Both evaluators were blinded to the group assignment of the calves and the results of each imaging study. The CR and MDCT studies were evaluated for diagnostic quality and graded as poor, acceptable, and excellent. The imaging studies were graded as excellent if no motion artifacts were present, as acceptable if motion was present to a degree that did not hinder evaluation of the lung parenchyma and pleural surface, or as poor if the study had to be repeated due to extensive motion causing a non-diagnostic study.

Additionally, on CT images the maximum height and length of the right and left lung were measured as well as the maximum tracheal diameter in the mid thorax and at the level just cranial of the tracheal bifurcation. Furthermore, the attenuation of the lung parenchyma was measured in pre- and post-contrast agent injection images at the ventral, mid, and dorsal levels by drawing a circular region of interest (ROI) of $0.3 \mathrm{~cm}$ in diameter encompassing lung parenchyma at each level and intercostal space to measure the average Hounsfield units (HU) in this drawn area. Pre- and post-contrast agent injection images were lined up with each other, so that measurements were made in the same anatomic area. No larger vessels or bronchi were included in the ROIs.

For evaluation of the lung in each imaging modality, the thorax was divided in four quadrants (Figure 1). The quadrants were defined by a horizontal line running parallel to and at the level of the ventral tracheal margin for a dorsal-ventral delineation, and by a vertical line at the level of and parallel to the caudal margin of the fifth rib for a cranial-caudal delineation. The resulting quadrants of the thorax were craniodorsal (CrD), cranioventral $(\mathrm{CrV})$, caudodorsal $(\mathrm{CdD})$, and caudoventral $(\mathrm{CdV})$. Within each quadrant, alterations of the lung parenchyma and airways were assessed by describing the predominant abnormal pulmonary pattern using the following criteria: presence of prominent and/or thickened bronchial walls (bronchial), a diffuse increased opacity of the lung parenchyma causing loss of definition of the vascular structures (unstructured interstitial), a soft tissue opacity of the lung with the presence of air bronchograms (alveolar with air bronchograms) completely obscuring identification of the vascular structures, borders of the heart or diaphragm, a focal soft tissue opacity in the lung without the presence of air bronchograms (alveolar without air bronchograms), which is completely obscuring identification of other structures. The presence of one or more soft tissue opacity structures measuring up to $3 \mathrm{~cm}$ in diameter (nodular), and no abnormality of the pulmonary parenchyma was noted (normal).

In each quadrant of the thorax, an estimate of the percentage of abnormal lung parenchyma was made based on the presence of any pathologic pattern being identified regardless of severity. For example, if $50 \%$ of a region was abnormal due to an unstructured interstitial pattern and $50 \%$ due to an alveolar pattern with air bronchograms, then this region would be listed as 100\% abnormal. If an entire region of lung was considered normal, the region was labeled as $0 \%$ to indicate absence of disease.

Furthermore, the presence of pleural fluid was recorded as a yes/no result. The degree of pleural fluid was noted as: within the

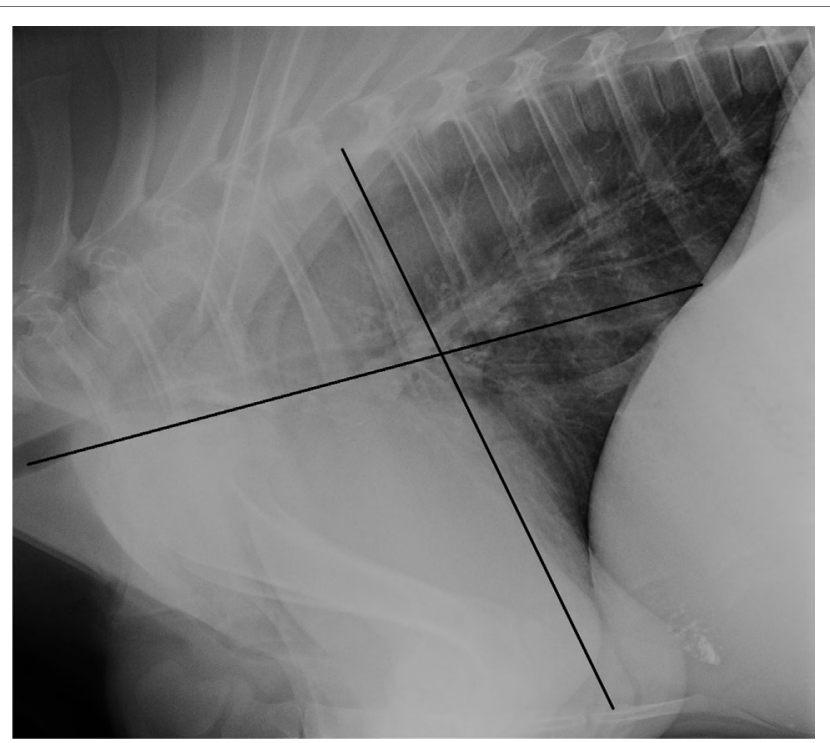

FIGURE 1 | Division of the thorax into four quadrants for pulmonary pattern and severity identification is illustrated on a lateral radiograph of the thorax of a 69-day-old Jersey heifer calf. The line parallel with the ventral aspect of the trachea divides the thorax into a dorsal and ventral half. The line parallel with the caudal border of the 5th rib divides the thorax into a cranial and caudal half. 
limits of the ventral third of the thorax (mild), within the limits of the mid third of the thorax (moderate), and extending to the dorsal third of the thorax (severe).

Additionally, the presence of visceral and parietal pleural thickening was recorded as a yes/no answer. The degree of pleural thickening was graded as: less than three focal areas of thickening noted (mild), between 4 and 6 areas of focal pleural thickening noted (moderate), and more than 7 areas of focal pleural thickening noted (severe).

\section{Pathology and Microbiology}

Prior to gross necropsy, nasopharyngeal swabs were collected in each calf for Mycoplasma culture and speciation, and M. bovis polymerase chain reaction (PCR). During necropsy, lung tissue was collected from various lung regions and submitted for Mycoplasma culture and speciation, $M$. bovis PCR, and aerobic bacterial culture and sensitivity. Bacteriology and molecular biology diagnostic evaluations were performed at the Oregon State Veterinary Diagnostic Laboratory (Corvallis, OR, USA). Mycoplasma speciation was performed by fluorescent antibody microscopy at the Wisconsin Veterinary Diagnostic Laboratory (Madison, WI, USA). Samples of all major organ tissues were harvested for histopathology.

\section{Statistical Analysis}

Statistical analysis was performed with commercial software (GraphPad Prism, version 6.04, GraphPad Software Inc., La Jolla, CA, USA). Quantitative data were assessed for normality using the Kolmogorov-Smirnov normality test and reported as mean \pm SD when normally distributed and as median and range when not. For comparisons between disease groups, quantitative, normal distributed data were analyzed using a one-way ANOVA with Sidak's multiple comparisons test adjustment for group differences, not normal-distributed quantitative data were analyzed using a Wilcoxon rank sum test, and binary data were analyzed using Fisher's exact test.

For comparisons of imaging modalities within calf, comparisons of lung quarters within the same calf, and for comparison of evaluators within the same calf, multinomial categorical data were analyzed using a Wilcoxon matched pairs signed rank test and binary paired data were analyzed using McNemar's test. Categorical data with more than two categories (i.e., disease stage) were collapsed to binary data, if one category dominated the other categories. Inter- and intra-observer agreements were calculated of multinomial categorical data and statistically analyzed using kappa statistics. Statistical significance was set at $p<0.05$.

\section{RESULTS}

\section{Animals}

Calves in the first-time diagnosis group (group 1) and the shortterm chronic group (group 2$)$ were similar in age ( $40.7 \pm 16.2$ versus $47.3 \pm 13.9$ days) and respiratory scores (group $1-9.6 \pm 1.5$, range: $7-12$; group $2-8.3 \pm 1.6$, range $5-11$ ). Calves in the longterm chronic group (group 3 ) were older than calves in group 1 and 2 (63.4 \pm 19.3 days) and had statistically lower respiratory scores than group $1(7.6 \pm 1.8$, range: $4-11 ; p=0.03)$. Of the respiratory score criteria, only body temperature differed between groups 1 and 3 (group $1-40.1 \pm 0.4 \mathrm{C}$, group $3-38.7 \pm 0.7 \mathrm{C}$; $p<0.0001$ ). All group 1 calves had a body temperature of $39.5 \mathrm{C}$ or greater, whereas only 3 calves in group 2 and 1 calf in group 3 had a body temperature of $39.5 \mathrm{C}$ or greater.

All 30 calves selected for inclusion in the study survived the imaging procedures without the need for additional medical care or sedation reversal agents.

\section{Thoracic Computed Radiography (CR)}

All, but one radiographic study was performed with the calves standing. One calf was unwilling to stand, so the calf was positioned sternally with the forelimbs extended cranially and left-to-right lateral radiographs of the thorax were obtained. Two radiographs of the thorax were sufficient in all calves to ensure that the entire thorax was included within image collimation. All radiographic images were of acceptable to excellent quality and no repeat radiographic studies were required.

\section{Thoracic Multi-Detector Computed Tomography (MDCT)}

Pre-contrast studies were obtained in $100 \%$ of patients. Of the survey scans, all were deemed suitable for evaluation by both evaluators, without a marked degree of motion artifact to cause inhibition of pulmonary parenchyma evaluation.

Of the pre-contrast studies, $8 / 30(26.7 \%)$ cases had negligible motion artifact, 17/30 (56.7\%) cases had a mild amount of motion artifact causing mild loss of distinction of the margins of the tertiary bronchi, and 5/30 (16.6\%) cases had a moderate amount of motion artifact causing complete loss of distinction of the tertiary bronchi and a mild loss of definition of the larger secondary bronchi. No pre-contrast studies had marked motion artifact.

Intravenous contrast medium administration failed for one case, in which the jugular vein catheter displaced when moving the animal and the contrast medium leaked into the perivascular space of the neck. Post-contrast images were successfully obtained in $97 \%$ of cases $(29 / 30)$. Of the 29 cases in which post-contrast images were available for evaluation, all MDCT scans were deemed suitable for evaluation.

All studies had some degree of respiratory motion induced slice mismatch between the pre- and post-contrast medium administration acquisitions. All post-contrast medium administration studies had a respiratory motion artifact, with $21 / 30$ (70\%) having mild motion, and 8/30 (26.7\%) having moderate motion artifact. A marked amount of respiratory motion artifact was seen in $1 / 30$ (3.3\%); however, the severity of the artifact was not to the degree that it would prevent diagnostic assessment of the images. In the case of extravasation of contrast medium from the jugular vein, the degree of motion artifact was unchanged between the pre- and post-contrast medium administration images.

The cranial border of the cranial lungs was at the level of the first rib in all but one calf, in which the lung extended mildly 
cranial to the first rib. The caudal border of the lungs ranged from the level of the 11-13th rib (average $12.0 \pm 0.7$ ). The length and height of the right and left lungs as well as the tracheal diameter were not significantly different in any of the groups. The differences were less than $1.1 \mathrm{~cm}$ in right and left lung height and length, and less than $0.1 \mathrm{~cm}$ in the tracheal diameter between the three groups.

The lung parenchymal attenuation averaged throughout all lung lobes before and after intravenous iodinated contrast agent injection was not statistically different between the groups (Table 1). The average of contrast enhancement throughout all lung lobes was not statistically different between the groups and averaged in group $1=32.7 \mathrm{HU}$, in group $2=24.6 \mathrm{HU}$ and in group $3=30.4 \mathrm{HU}$. The maximum difference between before and after intravenous iodinated contrast agent injection images averaged $54.3 \mathrm{HU}$ in group 1, 49.3 HU in group 2, and 52.8 HU in group 3. The average lung attenuation in the $\mathrm{CrV}$ aspect of the lung lobes $(-140 \pm 290.7 \mathrm{HU}$, range -828.0 to $64.3 \mathrm{HU})$ was higher than in the $\mathrm{CdD}$ aspect of the lung lobes $(-693.7 \pm 121.0 \mathrm{HU}$, range -851.4 to $26.2 \mathrm{HU})$.

\section{Comparison of Thoracic Computed Radiography (CR) and Computed Tomography (CT) for Pulmonary Disease Diagnosis}

Both evaluators detected abnormal lung patterns, consistent with pulmonary disease, in all 30 calves with CT. Using the CT diagnosis of the more experienced evaluator as gold standard, the more experienced evaluator (SSV) also detected abnormal lung patterns in all 30 calves with CR (Figures 2-4). This was repeated when only the $\mathrm{CrV}$ quadrant was assessed, in which all patients had disease identified on both CT and CR. Using CR, the less experienced evaluator (JF) correctly identified 27 of 30 calves with abnormal lung patterns, and the three misidentified calves were chronic disease groups (two short-term and one long-term). Differences in abnormal lung detection between imaging modalities were not statistically significant $(p=0.25)$.

The severity of pulmonary disease diagnosis was evaluated based on lung pattern, for which alveolar was considered the most severe pulmonary disease pattern. The more experienced

TABLE 1 | Summary of the average \pm SD [range] lung attenuation in Hounsfield units (HU) pre- and post-intravenous iodinated contrast medium administration and the difference in attenuation between the pre- and post-intravenous iodinated contrast medium administration images sorted by group.

\begin{tabular}{|c|c|c|c|}
\hline Disease group & Attenuation (HU) pre-contrast & Attenuation (HU) post-contrast & $\begin{array}{l}\text { Difference in attenuation between } \\
\text { pre- and post-contrast }\end{array}$ \\
\hline Group 1-acute & $-456.8 \pm 81.9[-898.9-76.6]$ & $-424.1 \pm 82.3[-871.9-120.4]$ & $32.7 \pm 14.6[11.9-54.3]$ \\
\hline Group 2-short-term chronic & $-478.2 \pm 123.2[-851.5-123.2]$ & $-451.1 \pm 130.3[-836.2-125.8]$ & $24.5 \pm 17.8[-1.3-49.3]$ \\
\hline Group 3-long-term chronic & $-521.9 \pm 164.1[-869.1-164.0]$ & $-491.5 \pm 165.0[-841.2-108.1]$ & $30.4 \pm 11.2[16.1-52.9]$ \\
\hline
\end{tabular}

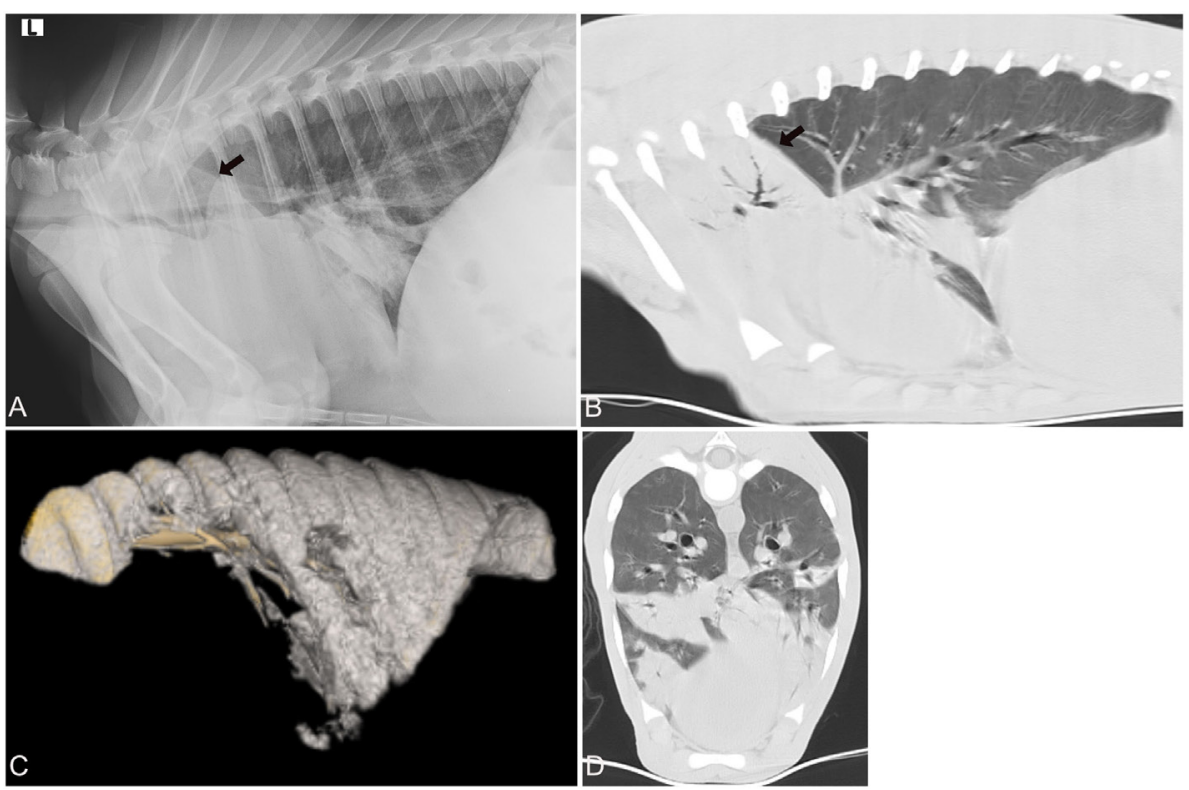

FIGURE 2 | Radiographic and computed tomography images of the thorax of a calf from group 1 (acute respiratory disease) with a respiratory score of 7 and body temperature of 40.5 C. Mycoplasma bovis was isolated and a bacterial coinfection was present. (A) Lateral radiograph and (B) sagittal reconstructed computed tomography (CT) image of the thorax in a lung window illustrating the alveolar lung pattern (black arrow) involving both cranial lung lobes, especially the right cranial lung lobe. (C) 3D reconstructed image of the air-filled lung. The cranial and cranioventral aspects of the lungs lack air-filling and are therefore not 3D reconstructed. (D) Transverse image of the thorax at the caudal aspect of the cardiac silhouette demonstrates the various regions in the lung with an alveolar lung pattern. 

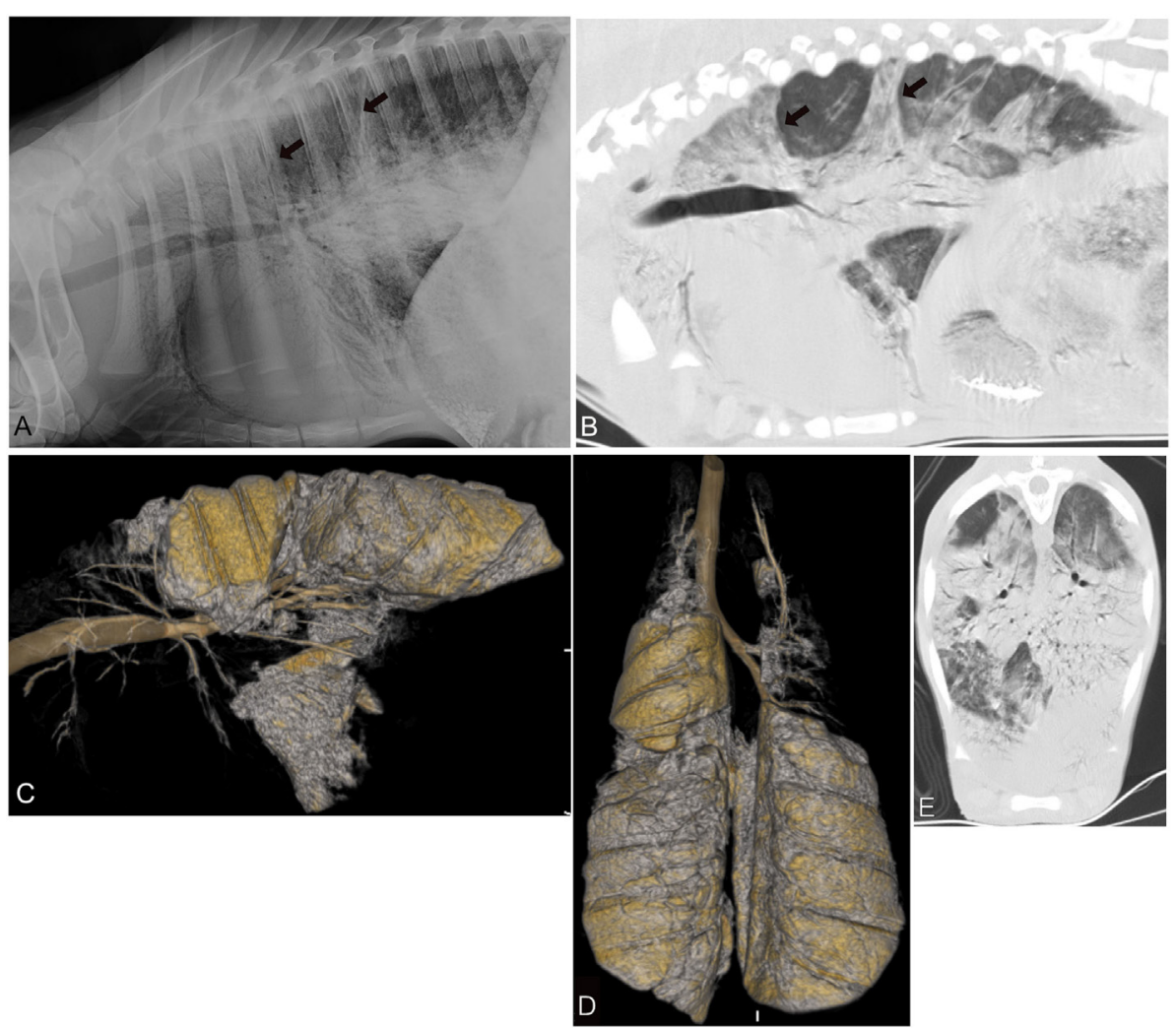

FIGURE 3 | Radiographic and computed tomography images of the thorax of a calf from group 3 (chronic-long term respiratory disease) with a respiratory score of 9 and body temperature of 39.1 C. (A) Lateral radiograph and (B) sagittal reconstructed computed tomography image of the thorax in a lung window illustrating the various areas of alveolar lung pattern (black arrow) involving the cranial aspects of the thorax most severely and to a lesser extent the caudodorsal aspects of the lungs. Three-dimensional (3D) reconstructed sagittal (C) and dorsal (D) image of the air-filled lung. The entire cranial and in part caudoventral aspects of the lungs lack air-filling and are therefore not 3D reconstructed. (E) Transverse image of the caudal thorax illustrating the various areas with a severe lung pattern occupying nearly all aspects of the lung parenchyma.

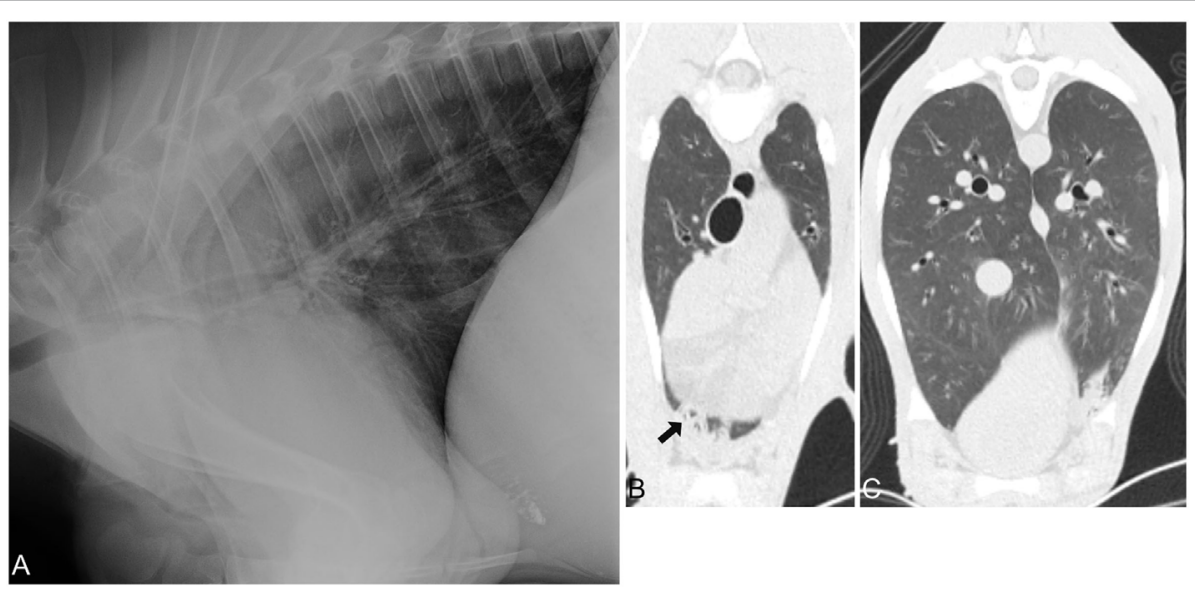

FIGURE 4 | Radiographic and computed tomography images of the thorax of a calf from group 3 (chronic-long term respiratory disease) with a respiratory score of 7 and body temperature of 38.1 C. No bacteria or Mycoplasma bovis were isolated. (A) Lateral radiograph of the thorax demonstrating minimal lung parenchymal changes of the most ventral and cranial aspects of the lungs. Most aspects of the lung are normally air filled. Transverse CT image of the cranial (B,C) caudal aspect of the lungs illustrating that only minimal alveolar changes are noted in the cranioventral aspects of the lungs, and no pathology was noted caudoventral and -dorsal. 
evaluator detected alveolar lung patterns in 29 calves using CT and correctly identified 28 of these calves as having an alveolar lung pattern and 1 calf as not $(n=1)$ using CR. The less experienced evaluator identified correctly 29 of 30 calves having either an alveolar lung pattern $(n=28)$ or not $(n=1)$ with CT and identified correctly 25 of these calves as having an alveolar lung pattern with CR. Four of the misdiagnosed calves were from the chronic disease groups (two short-term and two long-term), and one calf was from the acute disease group. Differences in severity assessment between imaging modalities were not statistically significant $(p=0.25)$.

Regardless of group, all of the calves had at least one lung quadrant with abnormal pulmonary patterns identified; none of the calves had a normal evaluation of all four quadrants of the lungs (Figure 2). In most calves (23 of 30 cases: group 1-nine calves, group 2-seven calves, group 3-seven calves) all four quadrants had an abnormal lung pattern (Table 2). Four calves (group 1-one calf, group 2-one calf, group 3-three calves) had three abnormal lung quadrants. Three calves (group 2-one calf, group 3-one calf) had two abnormal lung quadrants. The $\mathrm{CrD}$ and $\mathrm{CrV}$ quadrants had abnormal lung pattern in all calves (Figures 2-4). Of the two cranial quadrants, the ventral part was more severely affected, as alveolar lung patterns were observed only in the CrV but not in the CrD quadrant in 8 out of 30 calves $(p=0.01)$ by one evaluator (SSV) and 10 out of 30 calves $(p=0.009)$ by the other evaluator (JF). The three calves with two abnormal lung quadrants had normal CdV and CdD lung quadrants, whereas the four calves with three abnormal lung quadrants had normal CdD lung quadrants.

The less experienced evaluator (JF) was less likely to identify disease in the $\mathrm{CrD}$ quadrant than the more experienced evaluator (SSV) [80 versus $100 \%(p=0.01)$ ]. Similar trends were observed for the $\mathrm{CrV}$ quadrant (90 versus $100 \%$ ) and the $\mathrm{CdV}$ quadrant (77 versus 90\%). No clear trends were observed for the CdD quadrant, as five calves were identified as abnormal only by SSV and six calves were identified as abnormal only by JF. No group differences were detected, when comparing the acute with the chronically diseased calves.

Both evaluators had a moderate to high agreement between $\mathrm{CR}$ and $\mathrm{CT}$ for identifying diseased lung and lung pattern in the $\mathrm{CrV}$ quadrant with a Kappa of 0.70 and 0.96, respectively. Similarly, the inter-observer agreement for CR showed a high correlation with 0.97 and a moderate for CT with 0.70 for the $\mathrm{CrV}$ lung aspects (Table 3 ). All other areas had a lower inter-observer correlation. The least agreement between the two modalities was noted in the dorsal and caudal aspects of the lung (Table 4). Both evaluators identified the $\mathrm{CrV}$ quadrant as the most severely and extensively affected quadrant in all three groups. Alveolar pattern was frequently detected in the $\mathrm{CrV}$ quadrant on both CT (97\%) and CR (93\%), and the CrV quadrant had the highest area of alveolar pattern detected on both modalities ( $C T=95 \%, C R=93 \%$ ). This was followed by the $\mathrm{CrD}$ and $\mathrm{CdV}$ quadrants of the thorax. Of these two quadrants, the CdV was more extensively affected compared to the $\mathrm{CrD}$ quadrant. The least affected quadrant in all groups was the CdD quadrant using CR in which $10 \%$ of calves had an alveolar pattern, and this was similarly reported by both evaluators.

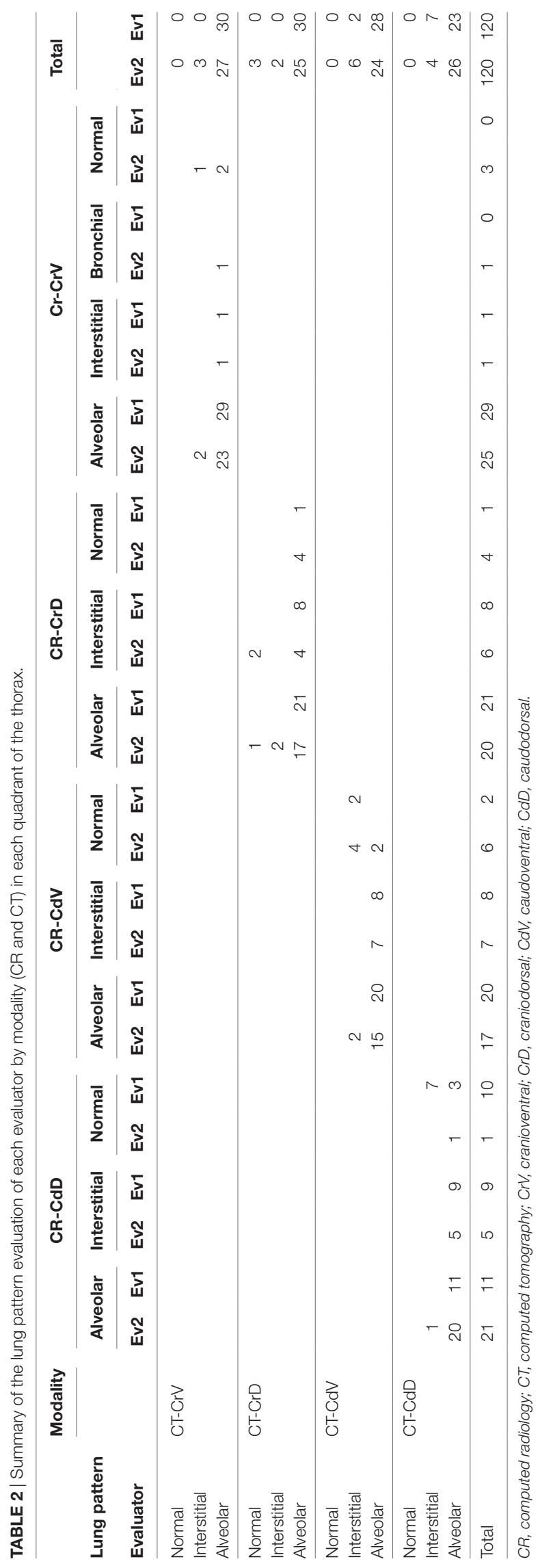


Generally, no significant differences were seen in the extent of affected lung between the three groups. The caudal quadrants tended to have a larger area of lung involved with longer disease duration. Otherwise, no strong correlation was noted between the lung pattern and length of disease course, or extent of diseased lung and length of disease course. In the earlier phase of disease (group 1) both evaluators had a better agreement in diagnosing lung pattern and extent of diseased lung. The largest disagreement between the evaluators was noted in the $\mathrm{CrD}, \mathrm{CdD}$, and $\mathrm{CdV}$ quadrants of the thorax in regards to lung pattern present. However, both evaluators were in close agreement that the lungs were extensively diseased.

No pleural fluid was noted in either imaging modality. A slightly higher number of cases with pleural thickening were noted using CT than CR (83\% using CT, compared to $76 \%$ using CR). The degree of pleural thickening ranged from mild to moderate and decreased mildly with chronicity of disease in all calves. In none of the cases, a diffuse or focal contrast enhancement of the pleura was noted. No statistically significant difference was noted between the acute and chronic diseases calves and between the individual groups.

No strong correlation was identified between the CR and CT imaging findings and the respiratory scores.

\section{Histopathology and Microbiology}

Similar to the imaging findings, all calves were histopathologically diagnosed with suppurative pneumonia (Table 5). Two calves (one each in group 1 and 2) were histopathologically additionally diagnosed with necrotizing pneumonia. The CrV aspects of the lungs were the most commonly and severely diseased areas of the lungs. The more dorsal lung aspects were either normal or had only mild changes suggestive of pneumonia. One calf in group 3 had mostly normal lung with only small areas of minimal consolidation. In one calf (group 1), histopathology and microbiology results of the lung tissue were unavailable.

TABLE 3 | Intra-observer agreement between radiography (CR) and computed tomography (CT) identifying a normal and abnormal lung patterns in the four quadrants of the thorax.

\begin{tabular}{|c|c|c|c|c|c|c|c|c|c|c|}
\hline \multirow{2}{*}{$\begin{array}{c}\text { Quadrant } \\
\text { Evaluator }\end{array}$} & \multicolumn{2}{|c|}{ CrV } & \multicolumn{2}{|c|}{ CrD } & \multicolumn{2}{|c|}{ CdV } & \multicolumn{2}{|c|}{ CdD } & \multicolumn{2}{|c|}{ Total } \\
\hline & Ev1 & Ev2 & Ev1 & Ev2 & Ev1 & Ev2 & Ev1 & Ev2 & Ev1 & Ev2 \\
\hline Agreement & 29 & 23 & 21 & 17 & 20 & 15 & 11 & 10 & 81 & 75 \\
\hline By chance & 1 & 7 & 9 & 13 & 10 & 15 & 19 & 20 & 39 & 45 \\
\hline Kappa & 0.97 & 0.70 & 0.57 & 0.24 & 0.50 & 0 & -0.73 & 0.5 & 0.52 & 0.4 \\
\hline
\end{tabular}

CrV, cranioventral; CrD, craniodorsal; CdV, caudoventral; CdD, caudodorsal.
Twenty-three calves (group 1-eight calves, group 2-seven calves, group 3-eight calves) had Mycoplasma isolated from the lung tissue (Table 5). Mycoplasma bovis was found in all but three positive cultures, in these three cultures M. bovirhinis was isolated. Additionally, in some calves other Mycoplasma species, including $M$. bovigenitalium (three calves) and $M$. alkalescens (three calves), were isolated. Pasteurella spp. was cultured from the lung tissue of 15 calves and all of these calves had co-infection with Mycoplasma. Only one Mycoplasma positive calf in group 3 had no bacteria recovered from the lung tissue. Only two calves in group 3 were negative for Mycoplasma in the lung tissue and had infections with Pasteurella multocida. In only one calf in group 3, in which minimal changes were seen on histopathology, no Mycoplasma or bacteria were isolated from the lung tissue.

\section{DISCUSSION}

In our study, radiographs of the calves were obtained in standing position, in all but one calf. All radiographs allowed evaluation of the CrV lung area and diagnosis of the area as diseased despite the summation with the forelimbs. In one study, calves were lifted from the floor to extend the front legs cranially (17), which required at least three persons holding the patient and pulling the legs away from thorax. In our study, usually only one person was holding the standing calf, which minimized the number of people close to the radiographic beam. This also decreased handling of the patient, which might further reduce the stress in the case of a respiratory distressed patient. Although intravenous sedation can be utilized to decrease the patient's stress and for chemical restraint, all calves enrolled in this study tolerated standing radiographs well while being awake. Furthermore, sedation may result in a decreased respiratory rate and effort and may therefore lead to incomplete aeration of the lung (atelectasis), which can be confused with lung disease such as pneumonia.

The intravenous sedation protocol utilized in this study provided a plane of sedation that was adequate for acquiring MDCT images of diagnostic quality without requiring repeat CT scanning. This is consistent with reports in small animals describing the use of MDCT in awake or sedated animal for the acquisition of pelvic CTs for trauma evaluation in dogs, the abdomen in dogs with acute abdominal signs, and studies of the cat respiratory tract for assessment of upper airway obstruction $(11-13,15)$. Although calves with respiratory disease commonly have tachypnea and cough as well as other signs of respiratory compromise, the plane of sedation provided by the sedation

TABLE 4 | Inter-observer agreement for radiography (CR) and computed tomography (CT) identifying a normal and abnormal lung patterns in the four quadrants of the thorax.

\begin{tabular}{|c|c|c|c|c|c|c|c|c|c|c|}
\hline Modality/quadrant & CR-CrV & CR-CrD & CR-CdV & CR-CdD & CT-CrV & CT-CrD & CT-CdV & CT-CdD & Total CR & Total CT \\
\hline Agreement & 29 & 21 & 20 & 11 & 23 & 17 & 15 & 20 & 87 & 69 \\
\hline By chance & 1 & 9 & 10 & 19 & 7 & 13 & 15 & 10 & 33 & 51 \\
\hline Kappa & 0.97 & 0.57 & 0.50 & -0.73 & 0.70 & 0.24 & 0 & 0.50 & 0.62 & 0.26 \\
\hline
\end{tabular}

CR, computed radiography; CT, computed tomography; CrV, cranioventral; CrD, craniodorsal; CdV, caudoventral; CdD, caudodorsal. 
protocol described herein allowed acquisition of CT images with a generally mild degree of respiratory motion, which did not preclude evaluation of the bronchial tree or pulmonary vasculature or parenchyma. In this study, the intraluminal contents of the bronchi could be evaluated to the level of the tertiary bronchi with ease in almost all cases. Although a degree of blurring of the smaller airways was appreciated in most of the patients, an evaluation of the Hounsfield units in the bronchial lumen could be made to enable identification of a lack of air-filling and abnormal bronchial intraluminal content.

The increased degree of respiratory motion noted in the postcontrast-enhanced MDCT images is likely due to the longer time post administration of sedation, but could in part also be due to the intravenous administration of a low-osmolar non-ionic iodinated contrast agent (iopamidol). It cannot be excluded that some of the calves had an immediate drug reaction leading to an increase in respiratory rate; however, currently no immediate adverse contrast agent reactions are reported in bovine species. For comparison, the percentage of immediate allergic drug reactions reported in human patients post injection of a low-osmolar non-ionic iodinated contrast agent is low ranging between 0.2 and $2.7 \%$. The human allergic drug response most frequently reported post intravenous low-osmolar non-ionic iodinated contrast agent injection included rash (85.3\%), itching sensation (58.8\%), nausea, and vomiting (6.8\%) followed by dyspnea, which was reported in $4.8 \%$ of cases in one study (18). No rash was noted in any of the calves post contrast agent injection. However, several of the signs noted as an immediate drug reaction in human patients could not be assessed in these calves. No anaphylactic responses secondary to the iopamidol contrast agent injection were noted in any of the calves in this study. Intravenous contrast medium was administered in this study to assess if the contrast enhancement pattern or degree could be utilized as an indicator of lung disease and disease duration; however, no statistically significant differences were seen in the enhancement between the three groups. Contrast medium enhancement can be utilized to identify if soft tissue attenuating material within the pleural space is due to fluid or vitalized tissue. In this study, no pleural fluid was seen in any patient. Had pleural fluid been present, contrast enhancement might have helped to provide assessment of which pleural layer, parietal or visceral, was thickened. The pleural thickening seen likely involved both pleural layers, but the visceral component was more extensive.

The $\mathrm{CrV}$ aspects of the lungs (Figures $\mathbf{2}$ and $\mathbf{3}$ ) were the most commonly and severely affected lung areas in both imaging modalities and on histopathology, which is similar to previous reports (5). The least affected quadrant was the CdD quadrant, and on $\mathrm{CR}$ the $\mathrm{CdD}$ quadrant had the lowest disease detection rate $(60 \%)$. As well, there was a higher variability of assessment between the examiners in the CdD quadrant suggesting that this region is more difficult to diagnose as abnormal, especially in the chronic disease cases. Examination of the CdD quadrant alone may be insufficient for detection of diseased patients. This is important to consider when imaging studies are performed as it has been suggested that radiographs of the CdD thorax are more easily obtained likely due to the lack of summation with the soft tissues from the forelimbs, and requiring less high exposure 
values, when compared to the $\mathrm{CrV}$ thorax using radiography (5). Given that radiography is a more accessible and on-farm available imaging modality for veterinarians, this is likely especially important to consider when attempting high quality thoracic radiographs using mobile radiographic units under field conditions where higher radiographic output setting to penetrate the $\mathrm{CrV}$ aspect of the thorax might not be available. Furthermore, obtaining radiographs of just the $\mathrm{CrV}$ aspect of the lungs might be sufficient to diagnose pneumonia as these areas were diagnosed with disease in more than $93 \%$ of the calves. However, none of the calves was diagnosed as completely normal by either of the evaluators using CR when the entire thorax was radiographed, which is consistent with the histopathology reports where all calves were diagnosed with pneumonia. Experience level impacted the assessment of severity of pulmonary disease, as the less experienced evaluator may underscore severity of disease based on imaging findings; this is unlikely to be clinically significant as the calves were still diagnosed with pulmonary disease requiring treatment. No significant difference between imaging modalities was found. Given these findings, CR and CT are likely equally effective in diagnosing acute and chronic pulmonary disease.

No strong correlation was seen between the clinical respiratory scores and the severity of disease on CR and CT. Mismatch between clinical severity and imaging severity is consistent with the concept of a lag between clinical disease and development of imaging abnormalities.

All calves were histopathologically diagnosed with suppurative pneumonia and two calves had additionally a necrotizing pneumonia. Only one calf had minimal lung changes identified histopathologically. Furthermore, abnormal lung patterns identified on imaging correlated well with the histopathology disease diagnosis. This has similarly been suggested for radiography in a previous experimental study (17).

The vast majority of the calves in the study had Mycoplasma isolated from lung tissues, and two thirds of those had a co-infection with Pasturella spp. This is similar to human studies, in which Mycoplasma is known to be a common agent for acute respiratory infection. Furthermore, it is known from human studies that Mycoplasma infection may precede and cause subsequent more severe infections with other viruses and bacteria due to immunosuppression and alteration of the normal respiratory flora by the Mycoplasma infections $(19,20)$. Only one Mycoplasma positive calf did not have a coinfection with other bacterial pathogens. It is possible that a fastidious organism was present but was not isolated. Future work such as this should include diagnostics with commercially available viral and bacterial bovine respiratory disease PCR assays.

As all the calves in the current study were diagnosed with pneumonia, an accuracy assessment could not be obtained. This limits the study in that we were unable to extrapolate the utility of the tests for a herd assessment tool. If animals without infectious pneumonia have pulmonary changes on imaging that are similar to findings associated with pneumonia, a false positive test result could occur. False positive diagnoses would lead to unnecessary antibiotic therapy, which may increase the risk of development of antibiotic resistant bacteria and treatment costs. We had expected less severe lung parenchymal changes in the calves enrolled in this study and assumed that more lung areas would have a normal lung pattern therefore allowing to better compare the imaging findings in normal and abnormal aspects of the lungs. However, it is important to note that imaging and histopathology findings were in agreement that the caudodorsal aspects of the lungs were the least affected or most normal aspects of the lungs suggesting that both imaging techniques will allow differentiating between normal and abnormal lung parenchyma.

A potential weakness of the study is the low number of evaluators in each group $(n=1)$; which has likely the largest effect when evaluating the differences between the evaluators. However, the comparison between both imaging modalities is likely less affected, especially considering that only minimal differences were noted.

In conclusion, both $\mathrm{CR}$ and sedated MDCT were equally effective in diagnosing acute and chronic pulmonary disease. Our results indicate than a less experienced evaluator can detect abnormal lung patterns with CR and MDCT; however, a less experienced evaluator may underscore disease severity. Although acute and chronic pulmonary disease severity can be assessed by both evaluators with both modalities. Furthermore, thoracic MDCT can safely be performed in sedated calves providing images of diagnostic quality. Combing clinical respiratory scores and imaging findings was inadequate for diagnosing chronicity of pneumonia in calves with naturally occurring pneumonia.

\section{ETHICS STATEMENT}

The study was approved by the Oregon State University Institutional Animal Care and Use Committee.

\section{AUTHOR CONTRIBUTIONS}

SS-V and JF made substantial contributions to the design of the study, data acquisition, analysis, and interpretation. JV and KP made substantial contributions to the design of the study, data acquisition, and interpretation. GB made substantial contributions to the design of the study, analysis, and interpretation. All authors agreed to the final version of the manuscript and have contributed to it.

\section{ACKNOWLEDGMENTS}

The authors thank Jason Wiest and Gregory Clausen for their technical assistance.

\section{FUNDING}

This study was supported by Oregon State University and the Department of Clinical Sciences Resident Research Program. 


\section{REFERENCES}

1. USDA. Dairy 2007, heifer calf health and management practices on U.S. dairy operations, 2007. USDA-APHIS-VS, CEAH. Fort Collins, CO (2007).

2. Wittum TE, Woollen NE, Perino LJ, Littledike ET. Relationships among treatment for respiratory tract disease, pulmonary lesions evident at slaughter, and rate of weight gain in feedlot cattle. J Am Vet Med Assoc (1996) 209(4): 814-8.

3. Adams EA, Buczinski S. Short communication: ultrasonographic assessment of lung consolidation postweaning and survival to the first lactation in dairy heifers. J Dairy Sci (2016) 99(2):1465-70. doi:10.3168/jds.2015-10260

4. Verschooten F, Oyaert W, Drubbel R. Radiographic diagnosis of lung diseases in cattle. Vet Radiol (1974) 15(1):49-59. doi:10.1111/j.1740-8261. 1974.tb00670.x

5. Bargai U, Pharr JW, Morgan JP. Bovine Radiology. Ames, IA: Iowa State University Press (1989).

6. Nykamp SG. The equine thorax. 6th ed. In: Thrall DE, editor. Textbook of Veterinary Diagnostic Radiology. St. Louis, MO: Elsevier (2013). p. 632-49.

7. Rizzi EB, Schinina V, Cristofaro M, Goletti D, Palmieri F, Bevilacqua N, et al. Detection of Pulmonary tuberculosis: comparing MR imaging with HRCT. BMC Infect Dis (2011) 11:243. doi:10.1186/1471-2334-11-243

8. Prather AB, Berry CR, Thrall DE. Use of radiography in combination with computed tomography for the assessment of noncardiac thoracic disease in the dog and cat. Vet Radiol Ultrasound (2005) 46(2):114-21. doi:10.1111/j.17408261.2005.00023. $\mathrm{x}$

9. Lubbers BV, Apley MD, Coetzee JF, Mosier DA, Biller DS, Mason DE, et al. Use of computed tomography to evaluate pathologic changes in the lungs of calves with experimentally induced respiratory tract disease. Am J Vet Res (2007) 68(11):1259-64. doi:10.2460/ajvr.68.11.1259

10. Henao-Guerrero N, Ricco C, Jones JC, Buechner-Maxwell V, Daniel GB. Comparison of four ventilatory protocols for computed tomography of the thorax in healthy cats. Am J Vet Res (2012) 73(5):646-53. doi:10.2460/ ajvr.73.5.646

11. Oliveira CR, Mitchell MA, O'Brien RT. Thoracic computed tomography in feline patients without use of chemical restraint. Vet Radiol Ultrasound (2011) 52(4):368-76. doi:10.1111/j.1740-8261.2011.01814.x

12. Lee K, Heng HG, Jeong J, Naughton JF, Rohleder JJ. Feasibility of computed tomography in awake dogs with traumatic pelvic fracture. Vet Radiol Ultrasound (2012) 53(4):412-6. doi:10.1111/j.1740-8261.2012.01939.x
13. Shanaman MM, Hartman SK, O'Brien RT. Feasibility for using dualphase contrast-enhanced multi-detector helical computed tomography to evaluate awake and sedated dogs with acute abdominal signs. Vet Radiol Ultrasound (2012) 53(6):605-12. doi:10.1111/j.1740-8261.2012. 01973.x

14. Lascola KM, O’Brien RT, Wilkins PA, Clark-Price Dvm SC, Hartman SK, Mitchell MA. Qualitative and quantitative interpretation of computed tomography of the lungs in healthy neonatal foals. Am J Vet Res (2013) 74(9):1239-46. doi:10.2460/ajvr.74.9.1239

15. Stadler K, O'Brien R. Computed tomography of nonanesthetized cats with upper airway obstruction. Vet Radiol Ultrasound (2013) 54(3):231-6. doi:10.1111/vru.12019

16. McGuirk SM. Disease management of dairy calves and heifers. Vet Clin North Am Food Anim Pract (2008) 24(1):139-53. doi:10.1016/j.cvfa.2007. 10.003

17. Tegtmeier C, Arnbjerg J. Evaluation of radiology as a tool to diagnose pulmonic lesions in calves, for example prior to experimental infection studies. $J$ Vet Med B Infect Dis Vet Public Health (2000) 47(3):229-34. doi:10.1046/ j.1439-0450.2000.00331.x

18. Kim SR, Lee JH, Park KH, Park HJ, Park JW. Varied incidence of immediate adverse reactions to low-osmolar non-ionic iodide radiocontrast media used in computed tomography. Clin Exp Allergy (2016) 47:106-12. doi:10.1111/ cea. 12803

19. Kashyap S, Sarkar M. Mycoplasma pneumonia: clinical features and management. Lung India (2010) 27(2):75-85. doi:10.4103/0970-2113. 63611

20. Parrott GL, Kinjo T, Fujita J. A compendium for Mycoplasma pneumoniae. Front Microbiol (2016) 7:513. doi:10.3389/fmicb.2016.00513

Conflict of Interest Statement: The authors declare that the research was conducted in the absence of any commercial or financial relationships that could be construed as a potential conflict of interest.

Copyright (c) 2017 Fowler, Stieger-Vanegas, Vanegas, Bobe and Poulsen. This is an open-access article distributed under the terms of the Creative Commons Attribution License (CC BY). The use, distribution or reproduction in other forums is permitted, provided the original author(s) or licensor are credited and that the original publication in this journal is cited, in accordance with accepted academic practice. No use, distribution or reproduction is permitted which does not comply with these terms. 